\title{
The Quest for Control Parameters in Motor Control May Be an Artifact of Experimental Paradigms
}

\section{Stan Gielen}

Latash gives a nice overview of the present state of the art in our understanding of the control and coordination of movements with multiple (abundant?) degrees of freedom. The overview is excellent regarding the behavioral studies. However, some references to neurophysiological studies, which provide support for the concepts pointed out by Latash, are lacking. For example, when he outlines future directions for research, Latash speaks about "the lack of tools to study patterns of control variables (such as time patterns of $\lambda$ for muscles and related control variables at the joint and whole-body levels) with sufficient accuracy." This is not completely correct as it is possible to investigate the activation of muscles in great detail using intramuscular wire electrodes, which measure the activity of single motor units. Van Zuylen et al. (1988) have given a detailed overview of the activation of arm muscles in the coordination of isometric flexion/extension and supination/pronation torques. These results show that the relative activation of muscles is very similar across subjects. Quite remarkably, the basic units for the coordination of isometric torques are not muscles, but subpopulations of motor units in a muscle, which each seem to have their own $\lambda$-value.

In a later study Tax et al. (1989) showed that the relative activation, and therefore also the relative contribution of arm muscles to force at the hand, is different when subjects exert the same force isometrically or while moving. Since the movements were very slow, any differences in muscle activation due to muscle mechanical contributions, such as the force-velocity relation ((Bolhuis et al., 1998) could be excluded. Also explanations for the different activation patterns based on variations simply due to co-contraction could be ruled out (Theeuwen et al., 1994). In terms of the equilibrium-point hypothesis, this result implies a different setting of the various $\lambda$-values for the arm muscles in isometric and isotonic movement tasks and convincingly demonstrates the existence of multiple muscle-activation synergies to obtain the same torque output. Therefore, these results provide strong evidence in favour of the hypothetical control scheme in Figure 7 of Latash (2010), which postulates covariation parameters (CV2), which allow for a broad range of "good performance" activation patterns with the same motor output.

In his overview, Latash emphasizes the principle of minimal muscle activation or minimal final action as a major principle for understanding human motor control. In my view there is little evidence in favour of this principle and, even worse,

Gielen is with Radboud University Nijmegen, Donders Centre for Brain, Cognition and Behavior. 
there is ample experimental evidence against this postulate. For example, m. biceps brachii makes a major contribution to supination torque and both the supinator muscle and $\mathrm{m}$. biceps brachii are activated during supination of the forearm (van Zuylen et al.,1988). Since m. biceps also contributes to flexion torque, activation of biceps for a pure supination torque requires activation of an extensor muscle to counteract the flexion contribution of $\mathrm{m}$. biceps. This explains the activation of the "pure extensor" triceps muscle during supination torques (van Zuylen et al., 1988). However, activation of biceps and triceps for supination is not compatible with the notion of minimization of muscle activation, as the latter would predict only activation of the supinator muscle.

The principle of minimal final action is not only contradicted by experimental findings, it may even be incompatible with the notion of high-level control. When we assume, that movements are controlled by setting high-level goals or system parameters, and that the movement output emerges given these parameter settings and the interaction with the environment, imposing minimization of muscle activation or final action seems futile as it tries to link high-level control directly to peripheral aspects of motor output regarding timing and amplitude of movements and forces. As Latash clearly points out, we have to distinguish between the control of motor acts and the coordination of movement and force. The coordination of movements or torques, and therefore, also the coordination of muscle activation patterns is the result of a control by the central nervous system and is different from the control itself.

This brings us to the question whether there are explicit control variables and, if so, what are they? Latash proposes that $\mathrm{R}$ (the referent position of the end point) and $\mathrm{C}$ (the apparent stiffness) might be the control variables. As shown by van Zuylen et al. (1988), Tax et al. (1989), and Theeuwen et al (1994) there are indeed multiple muscle synergies for the same condition of the end effector. However, these multiple muscle synergies do not differ by the amount of co-contraction (i.e., the parameter $\mathrm{C}$ ). This suggests that the main control parameters may not be $\mathrm{R}$ and $\mathrm{C}$.

The quest for control parameters in motor control has its roots in traditional control theory and in the nature of many experimental designs to investigate the human motor system. Regarding the latter, human motor performance is surprisingly flexible in dealing with many complex tasks in a wide range of environmental conditions. However, many experimental studies tested the motor system in artificially simple tasks, such as maintaining an isometric torque or to reach a particular end position. In such tasks, the instruction to the subject implicitly imposes control parameters and then it may be justified to ask whether and how these control parameters are represented in the nervous system. However, simple motor tasks are an exception in daily life, and complex movements may require different control principles (see e.g. Hermens et al. 2004). Therefore, the emphasis on control parameters may be an artefact of the simple experimental protocols

With regard to control theory, optimal control has been highly successful in engineering to control human-built systems. Typically, a system in engineering is designed for a particular purpose (for example, the central heating system in your house to maintain a pleasant temperature) and this purpose implies a unique set of control parameters. However, the human motor system is very different. It has not been designed for one particular function and therefore, it may not have one particular set of control parameters. Moreover, human subjects learn by trial and 
error. Based on feedback about the performance in a broad repertoire of movements, subjects modify their behaviour until they do a good job. Any high-level control parameters (if any!) are the result of learning a broad repertoire of movements, rather than the other way around that the performance is the result of high-level control parameters.

How then should we proceed? The best way to proceed may be to follow the lines of nature: start with a model of the motor control system, and try to use various learning theories (such Hebbian-based learning and reinforcement learning) to learn a broad repertoire of movement tasks (not just one simple task!). This approach requires a close interaction between theory and experiment where theoretical studies should be able to predict the muscle activation patterns and the resulting torques and movement trajectories. This is the proper scientific approach for any mature research field and brings a major challenge for experimentalists and theoreticians.

The work on control theory in motor control has mainly focussed on deterministic control. Harris and Wolpert (1998) were the first to incorporate (multiplicative) noise in theoretical modelling of motor control. A next step would be to incorporate other types of noise and, may be most important, uncertainty about the properties of the motor system and the environment. The proper way to do so is to expand the field of deterministic control to stochastic control (see e.g. Kappen (2005) and Todorov (2005)) and to partially-observable Markov decision processes (Kaebling et al., 1998). This will allow for control, where an adaptive system learns to characterize the time-dependent characteristics of human motor performance. The integration of theoretical approaches with neurophysiological and behavioural studies in humans and in non-human primates promises a challenging future for research on Motor Control.

\section{References}

Harris, C.M. and Wolpert, D.M. (1998) Signal-dependent noise determines motor planning. Nature 394: 780-784.

Hermens, F., Gielen, S. (2004) Posture-based or trajectory-based movement planning: a comparison of direct and indirect pointing movements. Experimental Brain Research 159: 340-348.

Kaebling L.P., Littman, M.L., Cassandra, A.R. (1998). Planning and acting in partially observable stochastic domains. Artificial Intelligence 101:99-134.

Kappen, H.J. (2005) A linear theory for control of non-linear stochastic systems. Physical Review Letters 95: 200201.

Tax, A.A.M., Denier van der Gon, J.J., Gielen, C.C.A.M. (1989) Differences in the activation of $\mathrm{m}$. biceps brachii in the control of slow isotonic movements and isometric contractions. Experimental Brain Research 76: 55-63.

Theeuwen, M., Gielen, C.C.A.M., Miller, L.E. (1994) The relative activation of muscles during isometric contractions and low-velocity movements against a load. Experimental Brain Research 101: 493 - 505.

Todorov E. (2005) Stochastic optimal control and estimation methods adapted to the noise characteristics of the sensorimotor system. Neural Computation 17: 1084-1108

van Bolhuis, B.M., Gielen, C.C.A.M., van Ingen Schenau, G.J. (1998). Activation patterns of mono- and bi-articular arm muscles as a function of force and movement direction of the wrist. Journal of Physiology 508: 313-324.

van Zuylen, E.J., Gielen, C.C.A.M., Denier van der Gon, J.J. (1988) Coordination and inhomogeneous activation of human arm muscles during isometric torques. Journal of Neurophysiology 60: 1523-1548. 